\title{
Primary instabilities in convective cells due to nonuniform heating
}

\author{
A. M. Mancho, ${ }^{1}$ H. Herrero, ${ }^{2}$ and J. Burguete ${ }^{1}$ \\ ${ }^{1}$ Departamento de Física y Matemática Aplicada, Facultad de Ciencias, Universidad de Navarra, 31080 Pamplona, Navarra, Spain \\ 2 Departamento de Matemáticas, Facultad de Ciencias Químicas, Universidad de Castilla-La Mancha, 13071 Ciudad Real, Spain
}

(Received 2 January 1997; revised manuscript received 11 April 1997)

\begin{abstract}
We study a convection problem in a container with a surface open to the air and heated by a long wire placed at the bottom. Coupled buoyancy and thermocapillarity effects are taken into account. A basic convective state appears as soon as a temperature gradient with horizontal component different from zero is applied. It consists of two big rolls that fill the convective cell and are parallel to the heater. A numerical solution allows us to determine this basic state. A linear stability analysis on this solution is carried out. For different values of the applied temperature gradient the basic rolls undergo a stationary bifurcation. The thresholds depend on the fluid properties, on the geometry of the heater, and on the heat exchange on the free surface. This confirms the results obtained in recent experiments. [S1063-651X(97)00609-0]

PACS number(s): 47.11.+j, 47.20.Dr, 47.20.Bp
\end{abstract}

\section{INTRODUCTION}

Convection provides one of the best physical systems out of equilibrium in which pattern formation and spatiotemporal complexities can be studied. Depending on the liquid and on the container different mechanisms (buoyancy, thermocapillarity, geometry, non-Boussinesq effects, local heating, and so on) can be selected to obtain several transitions and dynamic regimes $[1,2]$. For example, buoyancy and thermocapillarity effects are considered in the Bénard-Marangoni problem (BM). This instability arises in various important technological processes and has been the subject of a considerable amount of research work [3,4]. Typically, the heating is uniform through the bottom surface and the corresponding temperature gradient is vertical, but interest has recently arisen in applying the heating in a different way. For instance, heating on a lateral wall has been studied, mainly motivated by the need to understand the hydrodynamic aspects of crystal growth in a low-gravity environment [5]. We analyze localized heating on the bottom surface so that the temperature gradient is not purely vertical or horizontal. The study of localized effects in the general problem of turbulence is important in order to understand the processes involved at different scales because it is known that structures formed in a large scale are due to effects at a lower scale, i.e., localized effects, which traditionally are not taken into account but may generate significant differences in the real processes. In the present paper we study BM convection due to non-uniform heating which has a Gaussian shape in the transversal direction. This problem is related to the convection from a quasi-one-dimensional heater that has been analyzed experimentally in recent years [6-13]. In Ref. [6] when a slight temperature difference is applied between the heater and the top open surface, a pair of rolls appears without threshold. Their axes are parallel to the heater, i.e., perpendicular to the horizontal component of the temperature gradient. When the temperature difference is increased further, a bifurcation towards a structure of rolls perpendicular to the heater takes place. If the temperature difference is increased again, secondary oscillatory bifurcations appear and the resulting patterns have a wave number that is one- half of that of the stationary bifurcation. In Refs. [7-13] the basic motions are hardly visible and the primary motions correspond to some traveling waves; stationary patterns are not observed. Some theoretical results from these experiments are in Ref. [15]. In all these studies the heaters have low "heat capacity" and therefore it is conceivable that a coupling between the temperature distributions of the heater and the fluid exists [12]. This coupling has been avoided in Ref. [6], which corresponds to the situation treated here.

It could be thought that these problems are related to lateral heating, but experiences show different behaviors, because the instability observed in lateral heating consists mainly of stationary rolls that get unstable as hydrothermal waves. Their axes are perpendicular to the temperature gradient $[16,17]$. In one-dimensional (1D) heaters the instability of the basic state has the form of longitudinal rolls with their axes perpendicular to the heater, i.e., parallel to the horizontal component of the temperature gradient. There is a relevant theoretical paper by Smith and Davis [18] in which they study nonuniform lateral heating. The inhomogeneity consists of the application of a higher temperature near the bottom than near the top surface. They find longitudinal rolls like those observed with 1D heaters and those in our study.

The main aim of this paper is to study theoretically the effect produced by inhomogeneous heating, with a Gaussian shape in one direction, in a BM instability. The width of the Gaussian profile is measured by a parameter $\beta$ in such a way that large $\beta$ implies homogeneous heating and small $\beta$ means quasi-1D heating as described in Ref. [6], although in fact there are no experimental results accounting for a continuous change in $\beta$, because this parameter is difficult to control. We distinguish two situations depending on $\beta$ : the smooth inhomogeneity for large $\beta$ and the sharp inhomogeneity for small $\beta$. In both cases the curves of marginal stability are calculated for the primary bifurcation. The basic equations and the basic convective pattern are discussed in the second section of this article. The reference flow is obtained numerically because an analytical expression of this solution of the hydrodynamic equations does not exist. Section III is devoted to the linear stability analysis of this basic solution: the derivation of the equations and boundary con- 


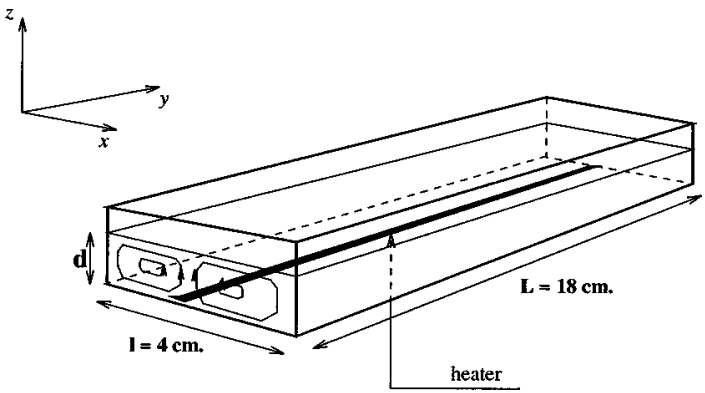

FIG. 1. Problem setup.

ditions (BC) for the perturbations and resolution of the corresponding eigenvalue problem. The results are presented distinguishing between the two cases explained previously. In Sec. IV we discuss the mechanisms of the instability, the main numerical results, and some conclusions. The numerical techniques used are explained in the Appendix.

\section{FORMULATION OF THE PROBLEM}

The physical situation that we consider is shown in Fig. 1. There is a horizontal fluid layer of depth $d$ ( $z$ coordinate) in a container of length $L$ ( $y$ coordinate) and width $l$ ( $x$ coordinate). As shown in the figure $L \gg l$ and for this reason $L$ can be considered to be infinite. The container has a rigid bottom plate and an upper surface open to the atmosphere. A heater is located in the middle of the bottom plate at $x=l / 2$, along the $y$ direction. The width of this heater is much smaller than the width of the container. The heater is at $T_{0}$, and the temperature of the environment is $T_{1}\left(<T_{0}\right)$. Then $\Delta T=T_{0}-T_{1}$ is the local difference of temperatures in the liquid layer just over the heater.

Starting from the general hydrodynamic problem several assumptions are convenient in order to perform a more tractable analysis. These are (1) the Oberbeck-Boussinesq approximation as usually assumed in convective problems. (2) The variation of the surface tension as a function of the temperature is approximated by $\sigma(T)=\sigma_{0}-\gamma\left(T-T_{0}\right)$, where $\sigma_{0}$ is the surface tension at temperature $T_{0}, \gamma$ is the constant rate of change of surface tension with temperature ( $\gamma$ is positive for most current liquids). (3) The length in the $y$ direction is considered infinite. (4) The free surface is assumed to be undeformable. This assumption is not strictly true, especially just over the 1D heater, but the deformation is very small as shown in the experiments of Kayser and Berg [7].

The system evolves according to the momentum balance equations and to the energy conservation principle. In the equations governing the system $u_{x}, u_{y}$, and $u_{z}$ denote the components of the velocity field $u$ of the fluid, $t$ the time, and $p$ the pressure. The spatial coordinates $(x, y, z)$ are represented by $r$. These variables are expressed in dimensionless form after rescaling in the following form: $r^{\prime}=r / d, \quad t^{\prime}=\kappa t / d^{2}, \quad u^{\prime}=d u / \kappa, \quad \Theta=\left(T-T_{1}\right) /(\Delta T), \quad p^{\prime}$ $=d^{2} p /\left(\rho_{0} \kappa \nu\right)$. (Here $\kappa$ is the thermal diffusivity, $\rho_{0}$ the mean density, and $\nu$ the kinematic viscosity of the liquid.) This normalization transforms the original spatial interval, $[-l / 2, l / 2] \times \mathbb{R} \times[0, d]$, into another one in which the limits are $[-\Gamma, \Gamma] \times \mathbb{R} \times[0,1]$. Here $\Gamma$ is the aspect ratio which is defined as $\Gamma=l /(2 d)$. Within Boussinesq's approximation, the governing dimensionless equations (the primes in the corresponding fields have been dropped) are the continuity equation

$$
\nabla \cdot u=0,
$$

the Navier-Stokes equation

$$
\partial_{t} u+(u \cdot \nabla) u=\operatorname{Pr}\left(-\nabla p+\Delta u+\frac{R \rho}{\alpha \rho_{0} \Delta T} e_{z}\right),
$$

the energy balance equation

$$
\partial_{t} \Theta+u \cdot \nabla \Theta=\Delta \Theta .
$$

$\nabla$ and $\Delta$ are the nabla and the Laplacian operators, respectively, $\alpha$ is the thermal expansion coefficient, $\rho$ the density, and $e_{z}$ is the unit vector in the $z$ direction. The following dimensionless numbers have been introduced:

$$
\operatorname{Pr}=\frac{\nu}{\kappa}, \quad R=\frac{g \alpha \Delta T d^{3}}{\kappa \nu},
$$

Pr is the Prandtl number, and $R$ the Rayleigh number, with $g$ the gravity. The Rayleigh number is representative of the buoyancy effect.

The above constants take the following values for the considered liquid (silicon oil):

$$
\begin{gathered}
\nu=5 \times 10^{-6} \mathrm{~m}^{2} / \mathrm{s}, \\
\kappa=1.24 \times 10^{-7} \mathrm{~m}^{2} / \mathrm{s} \\
\alpha=1.05 \times 10^{-3}{ }^{\circ} \mathrm{C}^{-1}, \\
\rho_{0}=910 \mathrm{~K} \mathrm{~g} / \mathrm{m}^{3} \\
\gamma=8.510^{-5} \mathrm{~N} \mathrm{~m}^{-1}{ }^{\circ} \mathrm{C}^{-1} .
\end{gathered}
$$

For this liquid $\operatorname{Pr}=40.32$, which is large enough to be considered as infinite.

\section{BASIC STATE}

When the wire heater is on, a temperature distribution on the bottom plate appears and as soon as the imposed gradient has a nonzero $x$ component, a convective motion sets in. In contrast to the classical BM problem with homogeneous heating on the bottom surface, the basic steady state in this case is not a conductive state, but a convective one. This motion has translational invariance in the $y$ direction and therefore the basic state has no dependence on the $y$ coordinate.

A very useful approach is to express the velocity field $u$ in terms of a potential as follows [19]: $u=\nabla \times \nabla \times \phi e_{z}$ $+\nabla \times \xi e_{z}$, where $\nabla \times \nabla \times \phi e_{z}$ is the poloidal part and $\nabla \times \xi e_{z}$ is the toroidal part, but if the fluid has an infinite Prandtl number, the equation for $\xi$ has only the solution $\xi=0$ and it is sufficient to consider the poloidal part. Therefore the final expression for the velocity field is $u=\left(\partial_{x} \partial_{z} \phi, \partial_{y} \partial_{z} \phi,-\Delta_{1} \phi\right)$, where $\Delta_{1}=\partial_{x}^{2}+\partial_{y}^{2}$. The translational symmetry in the $y$ direction for the basic state implies that all the derivatives in this coordinate are zero. So it is possible in this case to obtain a simpler expression for the 
velocity field. If we call $\psi=\partial_{x} \phi$, we have $u=\left(\partial_{z} \psi, 0, \partial_{x} \psi\right)$. This replacement allows us to reduce the order of the partial differential equations for the potential. Now the number of boundary conditions we have is appropriate to pose a correct problem.

For computational convenience, the coordinate transformations $x^{\prime}=x / \Gamma$ and $z^{\prime}=2 z-1$ have been performed (we drop the prime in the following), which transforms the domain in the $x-z$ directions into the square $[-1,1] \times[-1,1]$.

After eliminating the pressure and as $\operatorname{Pr}=\infty$, the momentum and energy equations in the steady state are

$$
\begin{gathered}
\frac{2}{\Gamma} \partial_{z} \psi \partial_{x} \Theta-\frac{2}{\Gamma} \partial_{x} \psi \partial_{z} \Theta=\Delta \Theta, \\
\Delta^{2} \psi-\frac{1}{\Gamma} R \partial_{x} \Theta=0,
\end{gathered}
$$

where $\Delta=\left(1 / \Gamma^{2}\right) \partial_{x}^{2}+4 \partial_{z}^{2}$.

\section{A. Boundary conditions}

As shown in experiments, there exists a Gaussian temperature distribution centered on the heater along the $x$ axis and at $z=-1$.

$$
\Theta(z=-1)=\exp \left[-(39 x)^{2} / \beta\right],
$$

where $-1 \leqslant x \leqslant 1$ and $\beta / 39^{2}$ is the width of the distribution. Therefore the values of $\beta$ taken in this paper correspond to the width of a Gaussian heating $\left(\exp \left[-x^{2} / \beta\right]\right)$ in a cell whose normalization is $[-39,39]$ in the $x$ direction. In order to keep the integer values of $\beta$ we do not rescale them.

The following BC ought to be fulfilled. $z=-1$,

$$
\begin{gathered}
\psi=0, \\
\partial_{z} \psi=0,
\end{gathered}
$$

(rigid bottom plate)

$$
\Theta=\exp \left[-(39 x)^{2} / \beta\right]
$$

for the heating on the bottom surface. $z=1$,

$$
B \Theta=-2 \partial_{z} \Theta .
$$

This condition accounts for the heat exchange on the free surface. $B$ is the Biot number which is defined by $B=h d / K$, where $h$ is the thermal surface conductance and $K$ is the thermal conductivity.

The surface tension due to temperature differences generates shear stresses on the liquid open surface. This condition may be expressed as [9]

$$
\nu \rho_{0}\left(\Delta_{1} u_{z}-\partial_{z}^{2} u_{z}\right)=-\Delta_{1} \sigma .
$$

The linear dependence of the surface tension $\sigma$ with the temperature allows us to write

$$
\nu \rho_{0}\left(\Delta_{1} u_{z}-\partial_{z}^{2} u_{z}\right)=-\partial_{T} \sigma \Delta_{1} T .
$$

After using a suitable rescaling and keeping in mind that there is no dependence on the $y$ coordinate, that condition transforms into

$$
\left(\frac{1}{\Gamma^{2}} \partial_{x}^{2}-4 \partial_{z}^{2}\right) u_{z}=-\frac{M}{\Gamma^{2}} \partial_{x}^{2} \Theta,
$$

where $M=(\gamma \Delta T d) /\left(\kappa \nu \rho_{0}\right)$ is the Marangoni number that describes the thermocapillarity effects. After assumption (4) there is no deflection on the free surface, and therefore $u_{z}=0$ on $z=1$ or $\partial_{x}^{2} u_{z}=0$ on $z=1$. After replacing $u_{z}=(1 / \Gamma) \partial_{x} \psi$ the final form for the viscous condition on the open free surface can be taken as

$$
-4 \partial_{z}^{2} \psi=\frac{M}{\Gamma} \partial_{x} \Theta
$$

This is the viscous condition on the open free surface.

Now we shall discuss the $\mathrm{BC}$ in the $x$ direction $(-1 \leqslant x \leqslant 1) . x= \pm 1$.

$$
\begin{gathered}
B_{s d} \Theta=-\frac{1}{\Gamma} \partial_{x} \Theta, \\
\psi=0, \\
\partial_{x} \psi=0,
\end{gathered}
$$

where $B_{s d}$ accounts for heat exchanges on the vertical walls, which are assumed to be insulating and therefore $B_{s d}$ is taken to be zero.

\section{B. Numerical results of the basic state}

We solved numerically Eqs. (5) and (6) with the boundary conditions (8)-(18) discussed previously and we found that the solution converges to a roll that fills the box with a flow rising at the center of the cell and moving towards the sidewalls. Here we only discuss the results obtained since the details about the numerical method are described in the Appendix.

As experimental results are mainly given in terms of $d$ and $\Delta T$, we use the liquid properties quoted in Ref. [6] to show our results in this representation. (Notice that only the kinematic viscosity changes from experiments in Ref. [6] $\left[5 c S\left(1 \mathrm{cS}=10^{-2} \mathrm{~cm}^{2} / s\right)\right]$ and that in Refs. $\left.[10,11](10 \mathrm{cS})\right)$. Some representative solutions are presented in Figs. 2 and 3. The temperature and the velocity profiles strongly depend on the heat exchanges across the open surface (measured by $h)$, on $\beta$, and, of course, on the applied difference of temperatures at the wire. For a fixed $\beta$, the larger the interchange of heat (large $h$ ), the larger the temperature difference across the layer at $x=0$ [compare Figs. 2(a) and 2(b)]. For a fixed $h$, by decreasing $\beta$ the temperature field and the streamlines become localized near $x=0$ [see Figs. 2 and 3(a) and 3(d)]. If we increase the applied difference of temperature at the origin, both fields expand in the cell [see Figs. 2 and 3(a) and 3(c)]. In short, small $\beta$ and $h$ generate small differences of temperature at the origin and therefore make the instability more difficult. 

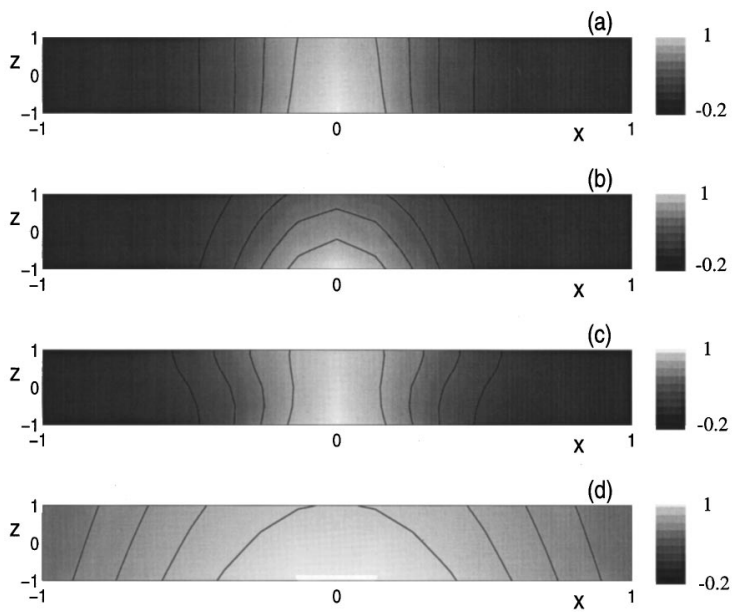

FIG. 2. Isotherms of the basic state before the instability for $d=1$ and different $\beta, h$ numbers and temperature differences $\Delta T_{c}$, the axes are $x$ and $z$. (a) $\beta=200, h=12.4\left(\mathrm{~W} / \mathrm{m}^{2}\right) /{ }^{\circ} \mathrm{C}$, and $\Delta T=1{ }^{\circ} \mathrm{C}$; (b) $\beta=200, h=124\left(\mathrm{~W} / \mathrm{m}^{2}\right) /{ }^{\circ} \mathrm{C}$ and $\Delta T=1{ }^{\circ} \mathrm{C}$; (c) $\beta=200, h=12.4\left(\mathrm{~W} / \mathrm{m}^{2}\right) /{ }^{\circ} \mathrm{C}$ and $\Delta T=7.57^{\circ} \mathrm{C}$; (d) $\beta=2500$, $h=12.4\left(\mathrm{~W} / \mathrm{m}^{2}\right) /{ }^{\circ} \mathrm{C}$, and $\Delta T=1{ }^{\circ} \mathrm{C}$.

\section{LINEAR STABILITY OF THE BASIC STATE}

To perform the linear stability analysis of the basic state calculated in the preceding section, it is perturbed with a vector field depending on the $x, y$, and $z$ coordinates, in a fully $3 \mathrm{D}$ analysis:

$$
\begin{gathered}
u_{b}(x, z)+\widetilde{u}(x, z) e^{\lambda t+i k y}, \\
\Theta_{b}(x, z)+\widetilde{\Theta}(x, z) e^{\lambda t+i k y} .
\end{gathered}
$$

In this expression the subscript $b$ indicates the corresponding quantity in the basic state and a tilde refers to perturbations. Notice that, as there are no boundary conditions in the $y$ direction, which is taken as infinite, it is possible to take the
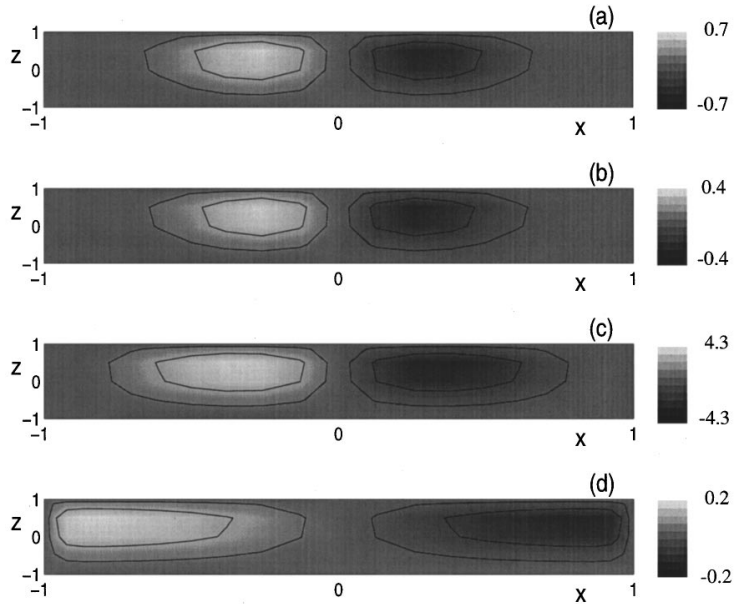

FIG. 3. Streamlines of the basic state for $d=1$ and different $\beta$, $h$ numbers, and temperature gradients, the axes are $x$ and $z$. (a) $\beta=200, h=12.4\left(\mathrm{~W} / \mathrm{m}^{2}\right) /{ }^{\circ} \mathrm{C}$ and $\Delta T=1{ }^{\circ} \mathrm{C} ; \quad$ (b) $\beta=200$, $h=124\left(\mathrm{~W} / \mathrm{m}^{2}\right) /{ }^{\circ} \mathrm{C}$, and $\Delta T=1{ }^{\circ} \mathrm{C} ; \quad$ (c) $\beta=200, h=12.4$ $\left(\mathrm{W} / \mathrm{m}^{2}\right) /{ }^{\circ} \mathrm{C}$, and $\Delta T=7.57{ }^{\circ} \mathrm{C}$; (d) $\beta=2500, h=12.4\left(\mathrm{~W} / \mathrm{m}^{2}\right) /{ }^{\circ} \mathrm{C}$ and $\Delta T=1{ }^{\circ} \mathrm{C}$.
Fourier modes in this direction, but the cell is finite in the $x$ and $z$ directions and the eigenfunctions are more difficult to calculate in this plane, so a development in series of Chebyshev polynomials was chosen in this case.

After replacing the expressions (19) and (20) into the hydrodynamic equations the following linear eigenvalue problem results:

$$
\begin{gathered}
\left(\lambda-\Delta+u_{x} \frac{\partial_{x}}{\Gamma}+2 u_{z} \partial_{z}\right) \Theta(x, z)+\partial_{x} \Theta \tilde{u}_{x}(x, z)+\partial_{z} \Theta \tilde{u}_{z}(x, z) \\
=0, \\
\Delta^{2} \tilde{u}_{z}(x, z)+R \Delta_{1} \Theta(x, z)=0 .
\end{gathered}
$$

Using the potential $\widetilde{\phi}$ instead of the velocity $\tilde{u}$ one arrives at

$$
\begin{gathered}
\left(\lambda-\Delta+u_{x} \frac{\partial_{x}}{\Gamma}+2 u_{z} \partial_{z}\right) \widetilde{\Theta}+\left(\frac{2}{\Gamma} \partial_{x} \Theta \partial_{x} \partial_{z}-\partial_{z} \Theta \Delta_{1}\right) \widetilde{\phi}=0 \\
-\Delta^{2} \Delta_{1} \widetilde{\phi}+R \Delta_{1} \widetilde{\Theta}=0
\end{gathered}
$$

The equations and boundary conditions result then in the following eigenvalue problem:

$$
\begin{aligned}
\left(\lambda-\Delta+u_{x} \frac{\partial_{x}}{\Gamma}+2 u_{z} \partial_{z}\right) \widetilde{\Theta}+\left(\frac{2}{\Gamma} \partial_{x} \Theta \partial_{x} \partial_{z}-\partial_{z} \Theta \Delta_{1}\right) \widetilde{\phi}=0 \\
-\Delta^{2} \Delta_{1} \widetilde{\phi}+R \Delta_{1} \widetilde{\Theta}=0 \\
-4 \partial_{z}^{2} \widetilde{\phi}-M \widetilde{\Theta}=0 \quad \text { at } \quad z=1 \\
\widetilde{\phi}=0 \quad \text { at } \quad z=-1,1 \\
\partial_{z} \widetilde{\phi}=0 \quad \text { at } \quad z=-1 \\
-2 \partial_{z} \widetilde{\Theta}-B \widetilde{\Theta}=0 \quad \text { at } \quad z=1 \\
\widetilde{\Theta}=0 \quad \text { at } \quad z=-1 \\
\widetilde{\phi}=0 \quad \text { at } \quad x=-1,1 \\
\partial_{x} \widetilde{\Theta}=0 \quad \text { at } \quad x=-1,1 \\
\partial_{x} \widetilde{\phi}=0 \quad \text { at } \quad x=-1,1 \\
\partial_{x}^{2} \widetilde{\phi}=0 \quad \text { at } \quad x=-1,1
\end{aligned}
$$

where the Laplacians are $\Delta=\left(1 / \Gamma^{2}\right) \partial_{x}^{2}+4 \partial_{z}^{2}-k^{2}$ and $\Delta_{1}=\left(1 / \Gamma^{2}\right) \partial_{x}^{2}-k^{2}$.

\section{A. Convergence of the numerical method for stability analysis}

The numerical method used in this section is explained in the Appendix. We discuss here the validity and convergence of the results obtained. In order to contrast the results provided by our algorithm we compare the temperature thresholds for the instability in the limit $\beta \rightarrow \infty$ with the very similar situation of classical BM convection in an infinite vessel for a fluid with the same physical properties as ours. Then 
TABLE I. Thresholds of instability for different orders in a pseudospectral approximation for finite and infinite vessels. The value of $h$ is $12.4 \mathrm{~W} \mathrm{~m}^{-2}{ }^{\circ} \mathrm{C}^{-1}$.

\begin{tabular}{lccccccc}
\hline \hline & \multicolumn{2}{c}{$11 \times 7$} & \multicolumn{2}{c}{$21 \times 9$} & \multicolumn{2}{c}{$\Gamma=\infty$} \\
\hline$d(\mathrm{~mm})$ & $k_{c}$ & $\Delta T_{c}$ & \multicolumn{2}{c}{$k_{c}$} & $\Delta T_{c}$ & $k_{c}$ & $\Delta T_{c}$ \\
1 & 2.05 & 5.97 & & 2.05 & 5.97 & 2.10 & 5.88 \\
\hline \hline
\end{tabular}

we draw up Table I, which shows no practical differences between the two algorithms, i.e., our numerical approach recovers known results.

In order to test convergence when we move away from known situations, we study the dependence of the thresholds of temperature $\left(\Delta T_{c}\right)$ for the instability of the basic state while the order of terms in the expansion of the perturbation fields increases. As the influence of increasing the order in the $z$-direction expansions $(M)$ is very small, we only consider the increasing order in $x$-direction expansions $(N)$. We consider that convergence is reached if differences of consecutive aproximations satisfy two conditions: these differences decrease for increasing order of the expansions and the difference between the $\Delta T_{c}$ calculated with consecutive approximations is less than $0.05{ }^{\circ} \mathrm{C}$. We think this is enough to provide an accurate value for $\Delta T_{c}$.

In Table II the values of the thresholds $\Delta T_{c}$ for $h=12.4\left(\mathrm{~W} / \mathrm{m}^{2}\right) /{ }^{\circ} \mathrm{C}$, two values of $\beta$, and increasing $N$ are quoted. These results satisfy the convergence conditions. It is clear also from the table that it is harder to obtain convergence when $\beta$ decreases, which means sharp inhomogeneity. For example, in Table III the same situation has been calculated for smaller values of $\beta$ and the results suggest that convergence has not been reached. This behavior is due to the chosen set of functions that is not adequate to perceive the details of a too localized boundary condition. In Tables $\mathrm{IV}$ and $\mathrm{V}$ and the same kind of calculations have been developed for increasing values of $h\left[124\left(\mathrm{~W} / \mathrm{m}^{2}\right) /{ }^{\circ} \mathrm{C}\right]$ and $1240 \mathrm{~W} / \mathrm{m}^{2} /{ }^{\circ} \mathrm{C}$ ), from which one can state that convergence improves if $h$ increases and convergence has been reached in the expansions considered, no matter the value of $\beta$.

\section{B. Stability results}

The results of the linear stability analysis show that the thresholds have a clear dependence on the shape of the as-

TABLE II. Critical temperature differences $\Delta T_{c}$ and wave numbers $k_{c}$ for $h=12.4\left(\mathrm{~W} / \mathrm{m}^{2}\right) /{ }^{\circ} \mathrm{C}$ and different values of $\beta$ increasing the number $N$ of Chebyshev polynomials used in the expansion of the perturbation fields.

\begin{tabular}{lcccc}
\hline \hline \multicolumn{5}{c}{$h=12.4\left(\mathrm{~W} / \mathrm{m}^{2}\right) /{ }^{\circ} \mathrm{C}$} \\
\\
$M \times N$ & $\Delta T_{c}$ & $\Delta T_{(N)-(N-2)}$ & $\Delta T_{c}$ & $\Delta=2500$ \\
& 6.72 & & 6.00 & \\
& 6.81 & 0.11 & 6.20 & 0.20 \\
$7 \times 9$ & 7.23 & & 6.16 & \\
$7 \times 11$ & 7.32 & 0.09 & 6.09 & -0.07 \\
$7 \times 19$ & 7.50 & & 6.12 & \\
$7 \times 21$ & 0.07 & 6.12 & 0.00 \\
$7 \times 25$ & 7.57 & 0 & \\
$7 \times 27$ & \multicolumn{5}{c}{} \\
\hline \hline
\end{tabular}

TABLE III. Critical temperature differences $\Delta T_{c}$ for $h=12.4\left(\mathrm{~W} / \mathrm{m}^{2}\right) /{ }^{\circ} \mathrm{C}$ and two different values of $\beta$, for which convergence is not good, increasing the number $N$ of Chebyshev polynomials used in the expansion of the perturbation fields.

\begin{tabular}{lcc}
\hline \hline \multicolumn{3}{c}{$h=12.4\left(\mathrm{~W} / \mathrm{m}^{2}\right) /{ }^{\circ} \mathrm{C}$} \\
\hline$M \times N$ & $\beta=100$ & $\beta=20$ \\
$7 \times 9$ & 6.95 & 7.05 \\
$7 \times 11$ & 7.26 & 7.70 \\
$7 \times 19$ & 8.22 & 11.16 \\
$7 \times 21$ & 8.46 & 12.09 \\
$7 \times 25$ & 9.04 & 13.98 \\
$7 \times 27$ & 9.34 & 14.36 \\
\hline \hline
\end{tabular}

sumed local heating, measured by $\beta$. From Fig. 4 and Table VI it is possible to conclude that the critical temperature for the stationary bifurcation decreases when $\beta$ increases so in the limit $\beta=\infty$ the instability threshold is minimum and it coincides with the usual BM problem [20]. In Fig. 5 we show the maximum eigenvalues of the stability analysis with $h=12.4\left(\mathrm{~W} / \mathrm{m}^{2}\right) /{ }^{\circ} \mathrm{C}$ for $\beta=200$ and $\beta=2500$ at $\Delta T_{c} \approx 6.12{ }^{\circ} \mathrm{C}$ which shows that in the former situation a higher $\Delta T_{c}\left(\Delta T_{c} \approx 7.57^{\circ} \mathrm{C}\right)$ is needed to reach instability. The value of $\beta$ affects not only the value of the critical temperature but also the value of the critical wave number. Figure 6 shows that the critical wave number increases for decreasing $\beta$.

The $h$ number on the top surface has a considerable influence. In Fig. 7(a) a curve $\Delta T_{c}$ vs $h$ for the stationary bifurcation and for a value of $\beta=2500$ is shown. There is a minimum threshold for medium $h$ while large and small thermal surface conductance inhibit instability. As for the critical wave number, it increases with $h$ [see Fig. 7(b)].

From Table VI we can appreciate the difference between the critical numbers for $\beta=2500$ (Fig. 7) and the ones obtained for $\beta=200$. We can see that the differences of temperature thresholds and critical wave numbers are larger for $\beta$ smaller. In this table the error in $k_{c}$ is \pm 0.05 .

The form of the growing perturbation for temperature and velocity fields is also provided by our analysis. Just after the instability the velocity and temperature in the cell are described by adding these perturbations times some small parameter to the basic state. For $\beta=200 h=12.4\left(\mathrm{~W} / \mathrm{m}^{2}\right) /{ }^{\circ} \mathrm{C}$ and $\Delta T=7.50^{\circ} \mathrm{C}$ Figs. 8 and 9 show the projections in the $y-z$ and $x-z$ planes, respectively, for the basic state, the growing perturbation, and the resultant temperature field.

In our stability analysis no oscillatory motions are obtained. On the other hand, the eigenvalue problem posed in

TABLE IV. Critical temperature differences $\Delta T_{c}$ for $h=1240\left(\mathrm{~W} / \mathrm{m}^{2}\right) /{ }^{\circ} \mathrm{C}$ and different values of $\beta$ increasing the number $N$ of Chebyshev polynomials used in the expansion of the perturbation fields.

\begin{tabular}{lcc}
\hline \multicolumn{3}{c}{$h=124\left(\mathrm{~W} / \mathrm{m}^{2}\right) /{ }^{\circ} \mathrm{C}$} \\
\hline$M \times N$ & $\beta=200$ & $\beta=2500$ \\
$7 \times 25$ & 1.58 & 1.53 \\
$7 \times 27$ & 1.58 & 1.53 \\
\hline \hline
\end{tabular}


TABLE V. Critical temperature differences $\Delta T_{c}$ for $h=1240 \mathrm{~W} / \mathrm{m}^{2} /{ }^{\circ} \mathrm{C}$ and different values of $\beta$ increasing the number $N$ of Chebyshev polynomials used in the expansion of the perturbation fields.

\begin{tabular}{lcc}
\hline \multicolumn{3}{c}{$h=1240\left(\mathrm{~W} / \mathrm{m}^{2}\right) /{ }^{\circ} \mathrm{C}$} \\
\hline$M \times N$ & $\beta=200$ & $\beta=2500$ \\
$7 \times 25$ & 2.96 & 2.89 \\
$7 \times 27$ & 2.96 & 2.89 \\
\hline \hline
\end{tabular}

Eq. (A3) does not have a self-adjoint operator $A$, so one could expect the appearance of complex eigenvalues [14]. However, for the considered parameters that does not happen. A similar situation occurs for the classical BénardMarangoni problem. The stability problem is defined in terms of a generalized eigenvalue problem in which there is not a self-adjoint operator. This is due to the presence of the Marangoni condition at the surface. However, only real eigenvalues appear in it. Now there is a similar situation even reinforced due to the presence of a nontrivial basic state that brings more factors that break self-adjointness in the operator of the problem. In spite of it no complex eigenvalues appear either.

\section{Comparison with the experimental results}

It is not possible to make a direct quantitative comparison because in the experiments several parameters are unknown, i.e., the $h$ and $\beta$ numbers. In Ref. [6] for $d=1$ a transition to longitudinal rolls appears for $\Delta T_{c} \approx 17^{\circ} \mathrm{C}$ and we obtain this transition for $\beta=200$ and $h=12.4 \quad\left(\mathrm{~W} / \mathrm{m}^{2}\right) /{ }^{\circ} \mathrm{C}$, $\Delta T_{c}=7.57^{\circ} \mathrm{C}$. As the thresholds increase when $\beta$ decreases, it is conceivable to think that it corresponds to a smaller value of $\beta$ that cannot be reached with our current numerical method.

As can be appreciated in Figs. 4 and 7(a), the results depend strongly on the $h$ number and on the $\beta$ number, which are unknown in these experiments, and theoretical results could fit with experimental ones for appropriate values of these parameters.

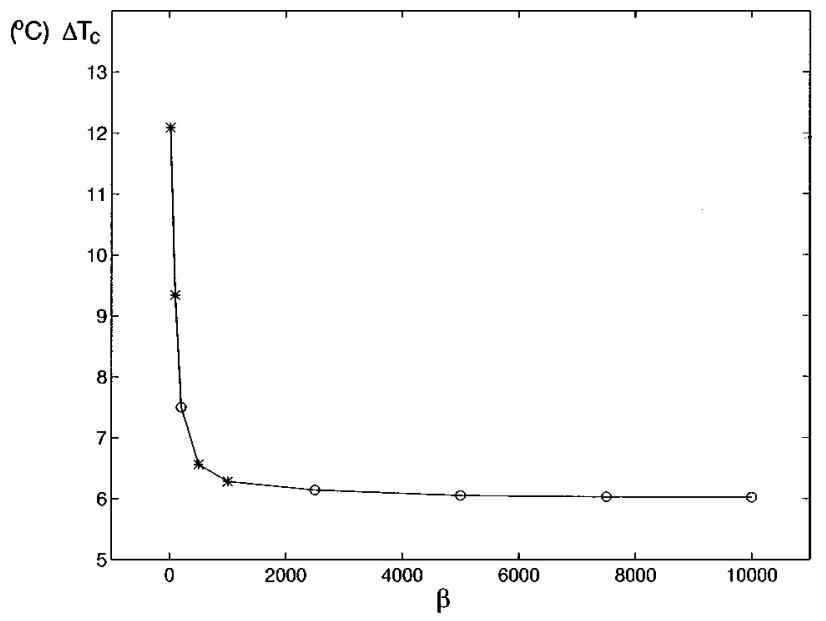

FIG. 4. Critical temperature thresholds $\Delta T_{c}$ as a function of the $\beta$ number for $h=12.4\left(\mathrm{~W} / \mathrm{m}^{2}\right) /{ }^{\circ} \mathrm{C}$. Circles correspond to values obtained with the method in which convergence was tested and asterisks correspond to values obtained without tested convergence.
TABLE VI. Critical temperature differences $\Delta T_{c}$ and wave numbers $k_{c}$ for the two significant values of $\beta$ and different values of $h$.

\begin{tabular}{lcccc}
\hline \hline$h\left(\mathrm{~W} / \mathrm{m}^{2} /{ }^{\circ} \mathrm{C}\right)$ & \multicolumn{2}{c}{$\beta=200$} & \multicolumn{2}{c}{$\beta=2500$} \\
\hline & $\Delta T_{c}$ & $k_{c}$ & $\Delta T_{c}$ & $k_{c}$ \\
12.4 & 7.50 & 2.20 & 6.09 & 2.05 \\
49.6 & 2.31 & 2.20 & 2.18 & 2.15 \\
86.8 & 1.75 & 2.25 & 1.69 & 2.25 \\
124 & 1.58 & 2.30 & 1.53 & 2.30 \\
217 & 1.50 & 2.40 & 1.46 & 2.40 \\
310 & 1.57 & 2.50 & 1.53 & 2.50 \\
403 & 1.68 & 2.55 & 1.64 & 2.55 \\
682 & 2.08 & 2.65 & 2.04 & 2.65 \\
961 & 2.52 & 2.75 & 2.46 & 2.70 \\
1240 & 2.96 & 2.85 & 2.89 & 2.75 \\
\hline \hline
\end{tabular}

Our results indicate that the stationary patterns are not interface motions which are excluded from the beginning [assumption (4) of Sec. II which is used in the derivation of the Marangoni equation in the open surface], but bulk motions. The mechanisms of the instability are buoyancy and thermocapillarity, but the patterns are not produced only on the surface, but influence the whole layer of fluid. In our opinion a quantitative explanation of the experiments in Refs. [10,11] would require a detailed knowledge of the full convective flow that appears before (or simultaneously to) the pattern of traveling waves. They seem to have a different physical origin, it could depend on interfacial motions which have been excluded in this analysis.

\section{CONCLUSIONS}

We performed a numerical analysis of convection due to an inhomogeneous heating, which has Gaussian shape in one direction, in a container with a surface open to the atmosphere. As the convective layer is open to the atmosphere, both buoyancy and thermocapillarity mechanisms contribute to the instability, although as depth is small, buoyancy effects are small also and the mechanism is mainly thermocap-

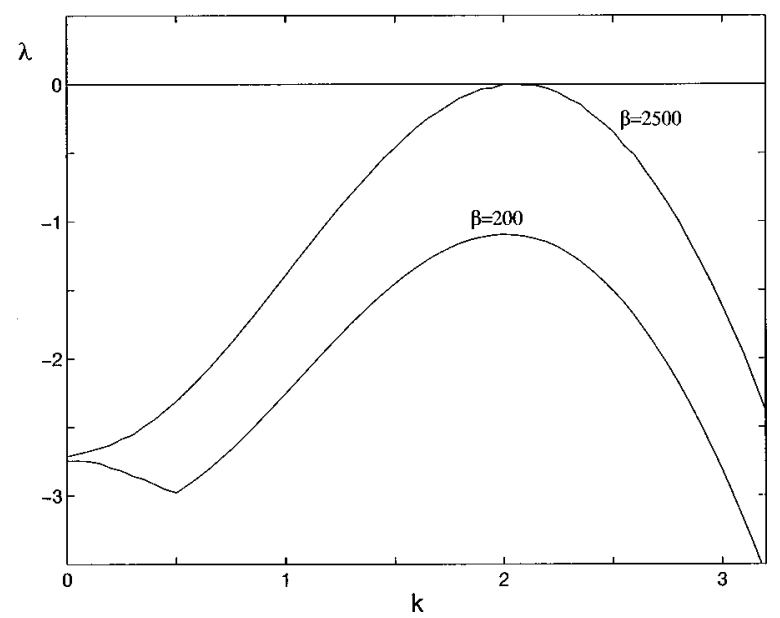

FIG. 5. The maximum eigenvalues $\lambda$ of the stability analysis for $h=12.4\left(\mathrm{~W} / \mathrm{m}^{2}\right) /{ }^{\circ} \mathrm{C}, \Delta T=6.12{ }^{\circ} \mathrm{C}$ and two different values of $\beta$ (200 and 2500). 


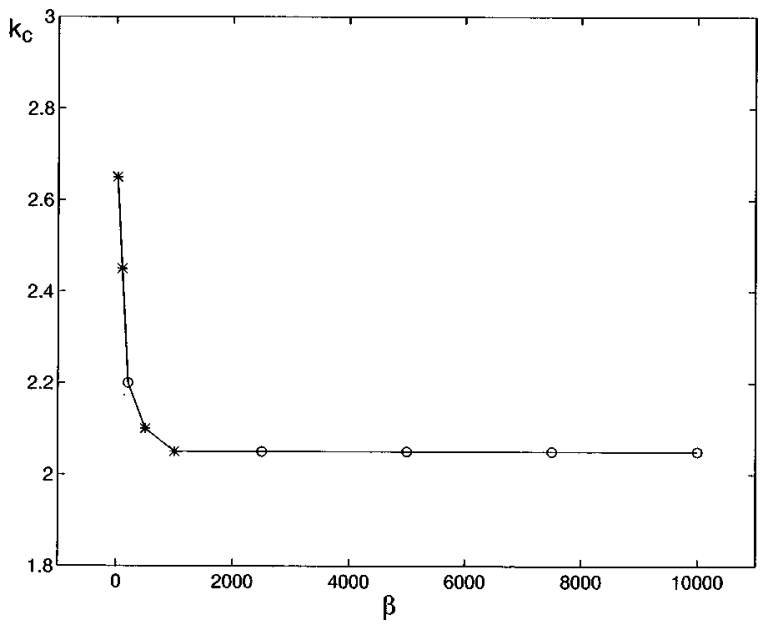

FIG. 6. Critical wave number $k_{c}$ as a function of the $\beta$ number for $h=12.4\left(\mathrm{~W} / \mathrm{m}^{2}\right) /{ }^{\circ} \mathrm{C}$. Circles correspond to values obtained with the method in which convergence was tested and asterisks correspond to values obtained without tested convergence.

illary. The basic state has been calculated numerically. It consists of a pair of rolls which go up over the heater and down near the sidewalls. It has been characterized for different shapes of the heating at the bottom plate (as measured by $\beta$ ) and for different values of the thermal surface conductance $h$.

An instability forming stationary rolls perpendicular to the basic ones is observed when the temperature difference at the origin is increased. In order to obtain this flow a linear stability analysis around the basic state has been performed. This implies a full 3D analysis of very heterogeneous fields which complicates the numerical analysis. The influence of the parameters affecting the problem is very different, thus the shape of the assumed Gaussian at the bottom layer of the fluid $(\beta)$ has an important influence on the critical difference of temperature thresholds and large $\beta$ favors the instability; the role played by the $h$ number at the top surface is very strong: the dependence of the critical temperature difference on $h$ is not monotonous and small and large $h$ numbers make instability more difficult than moderate ones. It also influences the critical wave number which increases monotonously with $h$. All the thresholds changing the $h$ number are increased for decreasing values of $\beta$.

Comparing these results with the events in BM convection with uniform heating, the BM instability is inhibited by the presence of a basic convective state. This is evident be-
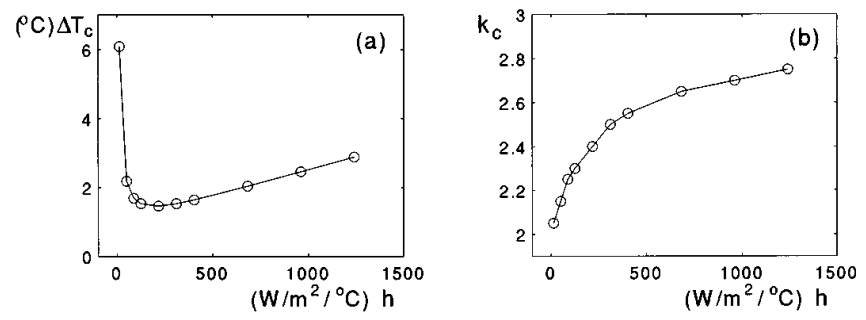

FIG. 7. (a) Critical temperature thresholds $\Delta T_{c}$ as a function of the $h$ number for $\beta=2500$ and (b) critical wave numbers $k_{c}$ as a function of the $h$ number for the same value of $\beta$. The circles correspond to the points calculated numerically.
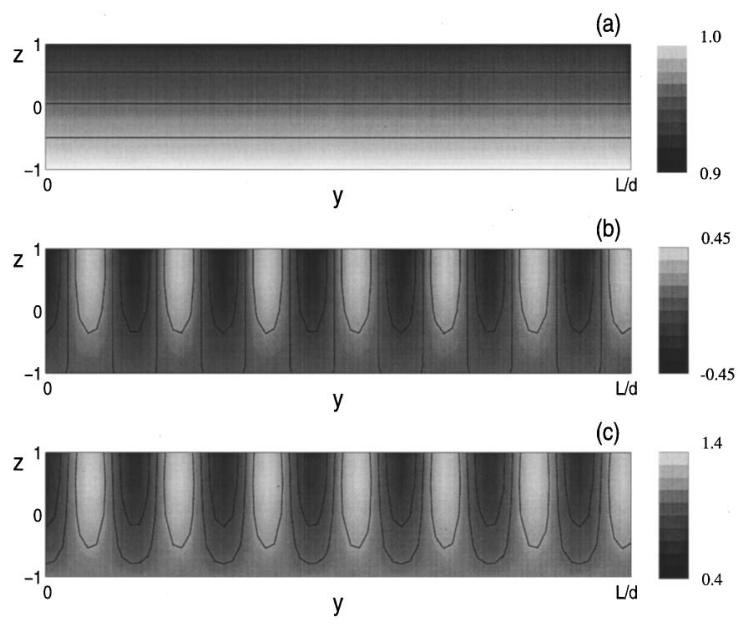

FIG. 8. Projection in the $y-z$ plane of the temperature field obtained for $\beta=200, h=12.4\left(\mathrm{~W} / \mathrm{m}^{2}\right) /{ }^{\circ} \mathrm{C}$, and $\Delta T=7.50{ }^{\circ} \mathrm{C}$. (a) The basic state; (b) the unstable eigenfunction times a small parameter $(\epsilon=0.5)$; (c) the temperature field after the instability. It is obtained by sumperimposing the plots (a) and (b).

cause the more the heating is localized the more the threshold for the stationary instability grows and in the limit without localization $(\beta=\infty)$ the thresholds coincide.

The agreement of our calculations and the experiments in Ref. [6] can be only qualitative since the results depend on the $h$ number and on the $\beta$ number, which are unknown in this experiment, and theoretical results could fit with experimental ones for appropriate values of these parameters. If this is compared to the experimental results in Refs. [10,11], they do not observe either the stationary patterns or the convective basic state, but only the oscillatory motions, but we think that the situation described in the present paper does not correspond to their situation owing to the coupling between the temperature distributions of the heater and the fluid and also to the fact that there can be interface motions. Our results reinforce the hypothesis that stationary bifurcating motions can be explained by bulk mechanisms and not by surface motions.
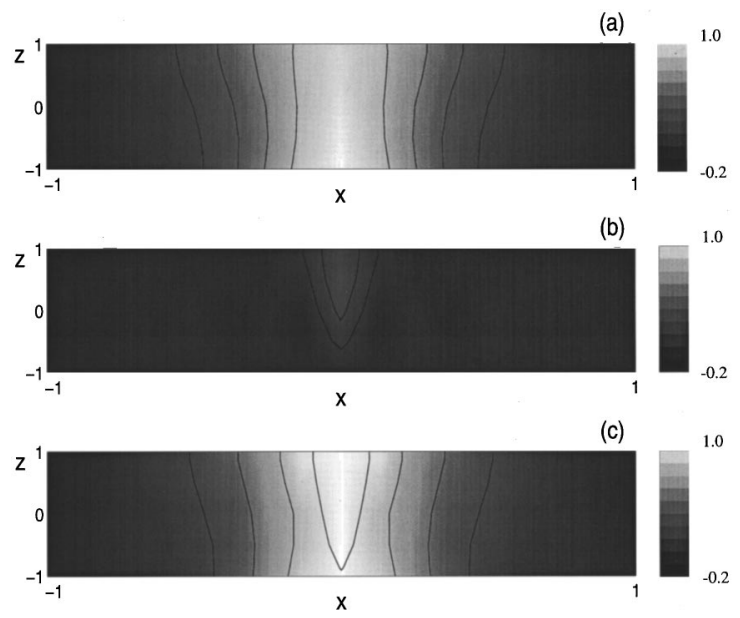

FIG. 9. Projection in the $x-z$ plane of the temperature field obtained for $\beta=200, h=12.4\left(\mathrm{~W} / \mathrm{m}^{2}\right) /{ }^{\circ} \mathrm{C}$, and $\Delta T=7.50{ }^{\circ} \mathrm{C}$. (a) The basic state; (b) the unstable eigenfunction times a small parameter ( $\epsilon=0.5$ ); (c) the temperature field after the instability. It is obtained by sumperimposing the plots (a) and (b). 


\section{ACKNOWLEDGMENTS}

We gratefully thank M. Bestehorn and I. Mercader for their help in the numerical analysis, and C. Pérez-García, A. Garcimartín, D. Maza, and H. Mancini for useful comments and discussions. H.H. thanks the Department of Applied Mathematics of the University Complutense of Madrid, where a part of this work was done. This work was partially supported by a Research Project PIUNA (University of Navarra) and DGICYT (Spanish Government) Grant No. PB950578 .

\section{APPENDIX}

\section{A. The numerical method for basic state}

We have solved numerically Eqs. (5) and (6) in a first step with a finite difference scheme to obtain an initial solution for a Chebyshev-collocation method that is the same as that used for the stability analysis calculations. To use this method we have linearized the equations expanding them around their solution at the previous time. This allows us to find the corrections to the successive approximations by solving linear systems. The criterion of convergence considered is that the difference between two consecutive steps at each point is smaller than $10^{-11}$.

\section{B. The numerical method for stability analysis}

The numerical method used to solve Eqs. (25)-(35) is a Chebyshev-collocation method. Following this approach, the perturbation fields $\widetilde{\Theta}$ and $\widetilde{\phi}$ are expanded in a truncated series of orthonormal Chebyshev polynomials $T_{n}(x) T_{m}(z)$ :

$$
\widetilde{\phi}(x, z)=\sum_{n=0}^{N-1} \sum_{m=0}^{M-1} a_{n m} T_{n}(x) T_{m}(z),
$$

$$
\widetilde{\Theta}(x, z)=\sum_{n=0}^{N-1} \sum_{m=0}^{M-1} b_{n m} T_{n}(x) T_{m}(z) .
$$

Expressions (A1) and (A2), are replaced into the linear equations, Eqs. (25) and (26), and boundary conditions Eqs. (27)-(35). The $N$ Gauss-Lobatto points $\left(x_{j}\right.$ $=\cos \{[-1+(j-1) /(N-1)] \pi\}, j=1, \ldots, N)$ in the $x$ axis, and the $M$ Gauss-Lobatto points $\left(z_{i}\right.$ $=\cos \{[-1+(i-1) /(M-1)] \pi\}, \quad i=1, \ldots, M)$ in the $z$ axis are calculated and the previous equations are evaluated at these points according to the following rules: Eq. (25) is evaluated at the points $\left(x_{j}, z_{i}\right)$ for $j=4, \ldots, N-3, \quad i=3, \ldots, M-2$, and Eq. (26) at the points for $j=2, \ldots, N-1, i=2, \ldots, M-1$. The boundary conditions at $z=1$, Eq. (27) and (28), are evaluated at $i=M, j=4, \ldots, N-3, \quad$ and Eq. (30) at $i=M, j=2, \ldots, N-1$. The boundary conditions at $z=-1$ are evaluated as follows: Eqs. (28) and (29) at $i=1, j=4, \ldots, N-3$ and Eq. (31) at $i=1, j=2, \ldots, N-1$. The boundary conditions at $x=1$, Eqs. (32)-(35) at $j=N, \quad i=1, \ldots, M$ and at $x=-1$ Eqs. (32)-(35) at $j=1, i=1, \ldots, M$.

There is a total of $2 \times N \times M$ algebraic equations with the same amount of unknowns $\left(a_{n m}\right.$ and $b_{n m}$, $n=0, \ldots, N-1 ; m=0, \ldots, M-1)$. If the coefficients of the unknowns which form the matrices $A$ and $B$ satisfy det $(A-\lambda B)=0$, a nontrivial solution of this linear homogeneous system exists. This condition generates a dispersion relation $\lambda \equiv \lambda\left(k, R, M, B, u_{b}, \Theta_{b}\right)$, equivalent to calculating directly the eigenvalues from the system

$$
A X=\lambda B X,
$$

where $X$ is the vector which contains the coefficients of the polynomials $a_{n m}$ and $b_{n m}$.
[1] S. Chandrasekhar, Hydrodynamic and Hydromagnetic Stability (Clarendon, Oxford, 1961).

[2] M. C. Cross and P.C. Hohenberg, Rev. Mod. Phys. 65, 851 (1993).

[3] S. H. Davis, Annu. Rev. Fluid Mech. 19, 403 (1987).

[4] A. Thess and S. A. Orszag, J. Fluid Mech. 283, 201 (1995).

[5] J. R. Carruthers, J. Cryst. Growth 42, 379 (1977).

[6] J. Burguete, H. Mancini, and C. Pérez-García, Europhys. Lett. 23, 401 (1993); D. Maza, J. Burguete, and H. Mancini, Int. J. Bifurcation Chaos Appl. Sci. Eng. 4, 1353 (1994); J. Burguete, H. Mancini, D. Maza, and C. Pérez-García, in Instabilities and Nonequilibrium Systems $V$, edited by E. Tirapegui and W. Zeller (Kluwer, Dordrecht, 1995); J. Burguete and H. Mancini (unpublished); J. Burguete, Ph.D. thesis, Universidad de Navarra, 1995 (unpublished).

[7] W. Kayser and J. Berg, J. Fluid Mech. 57, 739 (1973).

[8] R. Anthore, P. Flambet, P. Gouesbet, M. Rhazi, and M. Weil, Appl. Opt. 21, 2 (1982).

[9] J. K. Platten and J. C. Legros, Convection in Liquids (Springer, Berlin, 1984).
[10] M. Weill, M. Rhazi, and G. Gouesbet, Appl. Opt. 21, 1501 (1985).

[11] J. Vince and M. Dubois, Europhys. Lett. 20, 505 (1992).

[12] E. Ringuet, C. Roze, and G. Gouesbet, Phys. Rev. E 47, 1405 (1993); E. Ringuet, S. Meunier-Guttin-Cluzel, C. Roze, and G. Gouesbet, J. Phys. (France) II 4, 1243 (1994); E. Ringuet, Ph.D. thesis, Université de Rouen, 1994 (unpublished).

[13] D. Schwabe, R. Velten, and A. Scharmann, J. Cryst. Growth 99, 1258 (1990).

[14] E. Zauderer, Partial Differential Equations of Applied Mathematics (Wiley \& Sons, New York, 1989).

[15] N. D. Kazarinoff and J. S. Wilkowski, Phys. Fluids A 2, 1797 (1990).

[16] A. Garcimartín, Ph.D. thesis, Universidad de Navarra, 1994 (unpublished).

[17] F. Daviaud and J. M. Vince, Phys. Rev. E 48, 4432 (1993).

[18] M. K. Smith and S. H. Davis, J. Fluid Mech. 132, 119 (1983).

[19] F. H. Busse and E. W. Bolton, J. Fluid Mech. 146, 115 (1984).

[20] P. C. Dauby, Ph.D. thesis, Université de Liège, 1994 (unpublished). 